\title{
Structural MRI and Amyloid PET Imaging for Prediction of Conversion to Alzheimer's Disease in Patients with Mild Cognitive Impairment: A Meta-Analysis
}

\author{
Eun Hyun Seo ${ }^{1,2}$, Woon Yeong Park², and IL Han $\mathrm{Choo}^{2,3} \bowtie$ \\ ${ }^{1}$ Premedical Science, College of Medicine, Chosun University, Gwangju, Republic of Korea \\ ${ }^{2}$ National Research Center for Dementia, Chosun University, Gwangju, Republic of Korea \\ ${ }^{3}$ Department of Neuropsychiatry, School of Medicine, Chosun University, Chosun University Hospital, Gwangju, Republic of Korea
}

Objective The aim of this study was to explore the prognostic values of biomarkers of neurodegeneration as measured by magnetic resonance imaging (MRI) and amyloid burden as measured by amyloid positron emission tomography (PET) in predicting conversion to Alzheimer's disease $(\mathrm{AD})$ in patients with mild cognitive impairment (MCI).

Methods PubMed and EMBASE databases were searched for structural MRI or amyloid PET imaging studies published between January 2000 and July 2014 that reported conversion to AD in patients with MCI. Means and standard deviations or individual numbers of biomarkers with positive or negative status at baseline and corresponding numbers of patients who had progressed to $\mathrm{AD}$ at follow-up were retrieved from each study. The effect size of each biomarker was expressed as Hedges's g.

Results Twenty-four MRI studies and 8 amyloid PET imaging studies were retrieved. 674 of the 1741 participants (39\%) developed $\mathrm{AD}$. The effect size for predicting conversion to $\mathrm{AD}$ was 0.770 [95\% confidence interval (CI) $0.607-0.934$ ] for across $\mathrm{MRI}$ and 1.316 (95\% CI 0.920-1.412) for amyloid PET imaging ( $\mathrm{p}<0.001$ ). The effect size was 1.256 (95\% CI 0.902-1.609) for entorhinal cortex volume from MRI.

Conclusion Our study suggests that volumetric MRI measurement may be useful for the early detection of AD.

Psychiatry Investig 2017;14(2):205-215

Key Words Mild cognitive impairment, Alzheimer's disease, MRI, Meta-analysis.

\section{INTRODUCTION}

Alzheimer's disease $(\mathrm{AD})$ has an insidious onset with a slowly progressive course. Mild cognitive impairment (MCI) is an intermediate stage between cognitively normal and $\mathrm{AD}$. In particular, amnestic MCI is considered as a prodromal stage of $\mathrm{AD}$. MCI may therefore be a target for the prediction of who will develop $\mathrm{AD}$ prediction. Several markers during the prodromal MCI stage, including neuropsychological tests, have been proposed for the prediction of $\mathrm{AD}$. However, no

Received: October 11, 2015 Revised: May 15, 2016

Accepted: June 1, 2016 Available online: February 4, 2017

$\triangle$ Correspondence: IL Han Choo, MD, PhD

Department of Neuropsychiatry, School of Medicine, ChosunUniversity, Chosun University Hospital, 365 Pilmun-daero, Dong-gu, Gwangju 61452, Republic of Korea

Tel: +82-62-220-3104, Fax: +82-62-225-3659, E-mail: ilhan.choo@chosun.ac.kr (c) This is an Open Access article distributed under the terms of the Creative Commons Attribution Non-Commercial License (http://creativecommons.org/licenses/by$\mathrm{nc} / 4.0$ ) which permits unrestricted non-commercial use, distribution, and reproduction in any medium, provided the original work is properly cited. single study has included enough data to provide a reliable predictor of $\mathrm{AD}$.

The International Working Group (IWG) and National Institute on Aging Alzheimer's Association (NIA-AA) has proposed several biomarkers as diagnostic criteria for MCI, including cerebro spinal fluid (CSF) amyloid beta $(\mathrm{A} \beta)$ and tau, atrophy on MRI, glucose metabolism on [18F]-fluorodeoxyglucose positron emission tomography (FDG-PET) and fibrillar A $\beta$ burden on amyloid PET. ${ }^{1,2}$

FDG-PET has been suggested to be the strongest predictor for progression from $\mathrm{MCI}$ to $\mathrm{AD},{ }^{3}$ however, its high costs may limit its widespread adoption. A CSF assessment requires a lumbar puncture, which is regarded an invasive procedure, although it is widely implemented without problems in the elderly. In contrast, MRI is noninvasive, widely used in clinical settings and also could be easily performed. Atrophy measurements on MRI have been used to predict the progression to AD inpatients with MCI since the 1990s. Most studies have 
analyzed the predictive value of hippocampus volume as well as the volume of entorhinal cortex and other regions. Furthermore, prediction of progression to $\mathrm{AD}$ in patients with $\mathrm{MCI}$ based on MRI may be effective for clinical trials of $\mathrm{AD}$ prevention. However, no meta-analysis exploring the effect size of MRI volume for the prediction of progression to $\mathrm{AD}$ has been conducted among patients with MCI applying qualified diagnostic criteria.

The aim of this meta-analysis was to explore the prognostic values of biomarkers of neurodegeneration as measured by MRI measurement and amyloid burden as measured by amyloid $\mathrm{PET}$ imaging in predicting progression to $\mathrm{AD}$ in patients with MCI.

\section{METHODS}

\section{Search strategy}

Studies published between January 2000 and July 2014 were identified through a computer-based search of PubMed and EMBASE by the OS X Server system of iMac. In addition, the PubMed option 'Related Articles' was used, and the references of identified studies were reviewed to search for potentially relevant papers. For structural MRI the search strategy was performed with the following search terms: (magnetic resonance imaging OR MRI) AND (mild cognitive impairment) AND (Alzheimer OR Alzheimer's disease) AND (predict* OR conversion or progress*). For amyloid PET imaging, the search strategy was performed with the following search terms: (positron emission tomography OR PET) AND (amyloid imaging OR beta-amyloid) AND (mild cognitive impairment) AND (Alzheimer OR Alzheimer's disease) AND (predict* OR conversion or progress*).

\section{Selection criteria}

Three reviewers (WYP, EHS and IHC) independently reviewed the abstracts and titles identified by the database searches and together decided on the articles to be retrieved. These retrieved articles were reviewed by all three authors to identify suitable studies. Discrepancies were resolved by reaching a consensus. Reviewers were not blinded to the study authors and results. Inclusion criteria for relevant research studies were the following: 1) reported in English, included original data and published in a peer-reviewed journal; 2) longitudinal design consisting of patients who could be classified as having progressive MCI (MCI-P: patients with MCI who progressed to a clinical diagnosis of $\mathrm{AD}$ ) and stable MCI (MCI-S: patients with MCI who did not progress to AD); 3) diagnosis of MCI was made according to established criteria for MCI; ${ }^{4,5}$ 4) clinical diagnosis of $\mathrm{AD}$ was performed according to the National Institute of Neurological and Communicative Disorders and
Stroke and the Alzheimer's Disease and Related Disorders Association (NINCDS-ADRDA), ${ }^{6}$ and/or Diagnostic and Statistical Manual of Mental Disorders (DSM-IV); ${ }^{7}$ and 6) types of dementia other than $\mathrm{AD}$ were not present. In addition, if more than one study included an overlap in patient, then the largest and the most recent studies were included. If the criteria by which MCI was diagnosed were unavailable, the paper was included only after consensus from the three reviewers that the defined criteria were compatible with defined MCI criteria. The exclusion criteria were 1) studies in animals; 2) cross-sectional studies or clinical trials of medicine; 3 ) no or insufficient data; 4) reviews or symposium papers; 5) papers containing data that overlapped with another eligible study that was either larger or more recent; 6) progression status of MCI to $\mathrm{AD}$ was not provided.

\section{Data extraction}

The following information was extracted from the eligible papers: author names, year of publication, source of subjects, numbers of patients, age, sex, education, baseline Mini-Mental State Examination (MMSE), follow-up duration, region of interest for MRI imaging, radioisotope for amyloid PET imaging, and diagnostic criteria used for MCI and AD diagnoses.

Means and standard deviations or sensitivity and specificity values for patients with MCI-P and MCI-S at baseline were extracted by WYP and checked by IHC for each study. If a study fulfilled all inclusion criteria but did not report all relevant data, we contacted the authors to obtain supplementary data. Some authors did not respond to this request.

\section{Quality assessment}

Study quality was assessed independently by the same reviewers with the revised tool for the Quality Assessment of Diagnostic Accuracy Studies (QUADAS-2). ${ }^{8}$ Two blinded reviewers performed data extraction and QUADAS-2 scoring for each research study. A third reviewer served as a blinded expert in cases of disagreement.

\section{Data analysis}

The data were analyzed using Comprehensive Meta-Analysis software, version 2 (BiostatInc, USA). The measure of interest was the effect size Hedges's g, which is generally calculated as the difference between the group means divided by the pooled standard deviation. In the present analyses, Hedges's $g$ was calculated as the standardized difference at baseline between patients with MCI-P and patients with MCI-S. When the data were reported as sensitivity and specificity, Hedges's $g$ was calculated by the Comprehensive Meta-Analysis program (http://meta-analysis.com). The studies were tested for heterogeneity with the conventional Q-total tests and the $\mathrm{H}$ 
statistic. $^{9}$

The potential for publication bias was investigated using visual assessment of the funnel plot calculated by the Comprehensive Meta-Analysis software. Publication bias may lead to asymmetrical funnel plots. The presence of publication bias was analyzed by graphical inspection of funnel plots and by fail-safe analysis (Rosenthal's method).

\section{RESULTS}

\section{MCI conversion with Structural MRI}

The search process is presented in Figure 1A. A total of 1562 MRI papers were initially identified from PubMed and EMBASE after the first round of screening based on titles and abstracts. Of these, 1413 papers were excluded after review of titles and abstracts for the following reasons: investigations did not use structural MRI ( $\mathrm{n}=294)$, paper was a review or symposium ( $\mathrm{n}=543)$, paper did not address conversion from MCI to $\mathrm{AD}(\mathrm{n}=530)$, investigation was a clinical trials $(\mathrm{n}=20)$, paper was not published in English ( $\mathrm{n}=11)$, paper described a case report $(n=8)$ or investigation was in animals $(n=7)$. We assessed 149 full-text articles for eligibility. Of these, 125 papers were excluded for the following reasons: patients overlapped with those in a large or more recent study $(n=55)$, papers had no or insufficient data for meta-analysis $(n=53)$, enrollment was limited exclusively to demented or healthy control $(\mathrm{n}=5)$, investigations used a cross-sectional design ( $\mathrm{n}=5)$, investigations included results from a combination of other diagnostic modalities $(n=4)$, and clinical diagnosis did not follow the reference standard $(n=3)$. Twenty-four studies satisfied all inclusion and exclusion criteria (Table 1). ${ }^{10-33}$ We selected a randomeffects model to allow for both between- and within- study variation, which generates a more conservative result than a fixed-effects model. The weighted Hedges's $\mathrm{g}$ from the random effects models was 0.770 [ $95 \%$ confidence interval (CI) 0.607$0.934]$ across any volume of interest (VOI) MRI papers, 0.683 (95\% CI 0.494-0.873) for MRI studies of the hippocampus and 1.256 (95\% CI 0.902-1.609) for MRI studies of the entorhinal cortex (Figure 2A, C, D). A larger absolute value of the weighted Hedges's $g$ indicates a stronger effect size between MCI-P and MCI-S, which means the risk with which MCI progress to $\mathrm{AD}$ by MRI measurement. Tests for heterogeneity were not significant $(\mathrm{Q}=32.685, \mathrm{p}=0.087$, I-squared $=29.631$ for total MRI; $\mathrm{Q}=21.712, \mathrm{p}=0.116$, I-squared $=30.914$ for hippocampus; $\mathrm{Q}=0.501, \mathrm{p}=0.919$, I-squared $=0.000$ for entorhinal cortex).

\section{MCI conversion with amyloid PET}

A total of 183 amyloid PET papers were initially identified from PubMed and EMBASE (Figure 1B). Of these, $161 \mathrm{pa}-$ pers were excluded after review of titles and abstracts for the following reasons: investigations did not use amyloid PET $(n=22)$, paper was a review or symposium $(n=74)$, investigation did not address conversion from MCI to $\mathrm{AD}(\mathrm{n}=59)$, investigation was a clinical trials $(n=20)$, investigations included results from a combination of other diagnostic modalities $(n=2)$, papers were not reported in English papers $(n=11)$ orpaper described a case reports $(n=2)$. We assessed full-text articles for eligibility. Of these, 14 papers were excluded for the following reasons: patients overlapped with those in a larger or more recent study $(\mathrm{n}=11)$, papers had no or insufficient data for meta-analysis $(n=1)$, investigations used FDDNP-PET $(\mathrm{n}=1)$, and clinical diagnosis did not follow the reference standard $(n=1)$. Eight studies satisfied all inclusion and exclusion criteria (Table 2) ${ }^{29,32,34-39}$ Weighted Hedges's g from the random effects models was 1.316 (95\% CI 0.920-1.712) (Figure 2B). Tests for heterogeneity were not significant $(\mathrm{Q}=9.554, \mathrm{p}=0.215$, I-squared=26.735).

\section{Publication bias}

There was no clear indication of publication bias. The funnel plots were not skewed (Supplementary Figure 1 in the online-only Data Supplement). Rosenthal's fail-safe numbers were 746 for the any VOI MRI group, 290 for the hippocampus, 120 for the amyloid PET group and 46 for the entorhinal cortex.

\section{DISCUSSION}

This study aimed to explore the ability of MRI atrophy measures and amyloid PET to predict conversion to AD in patients with $\mathrm{MCI}$ across a number of published studies. Effect size, which was expressed as Hedges's g between patients with MCI-P and patients with MCI-S, was highest for amyloid burden as measured by amyloid PET, followed by MRI atrophy measures in the entorhinal cortex, and in any VOI cortex, and the hippocampus. These results suggest that amyloid PET is overall a better predictor of progression to $\mathrm{AD}$ from MCI than MRI atrophy measures. However, the entorhinal cortex atrophy measure on MRI is comparable in prediction value to amyloid PET.

There are few previous meta-analyses on longitudinal studies that have validated the progression from $\mathrm{MCI}$ to $\mathrm{AD}$. One previous meta-analysis reported that MRI atrophy measures had sensitivity of $72.8 \%$ and specificity of $81.0 \%$ for the prediction of conversion to $\mathrm{AD}{ }^{40}$ This meta-analysis included papers published only from 2000 to 2005 only, and a cognitively normal group was included in the baseline diagnostic criteria. In contrast, our study selected only patients with MCI as baseline diagnostic criteria and included papers over a broader range from 2000 to 2014. A meta-analysis by Zhang 

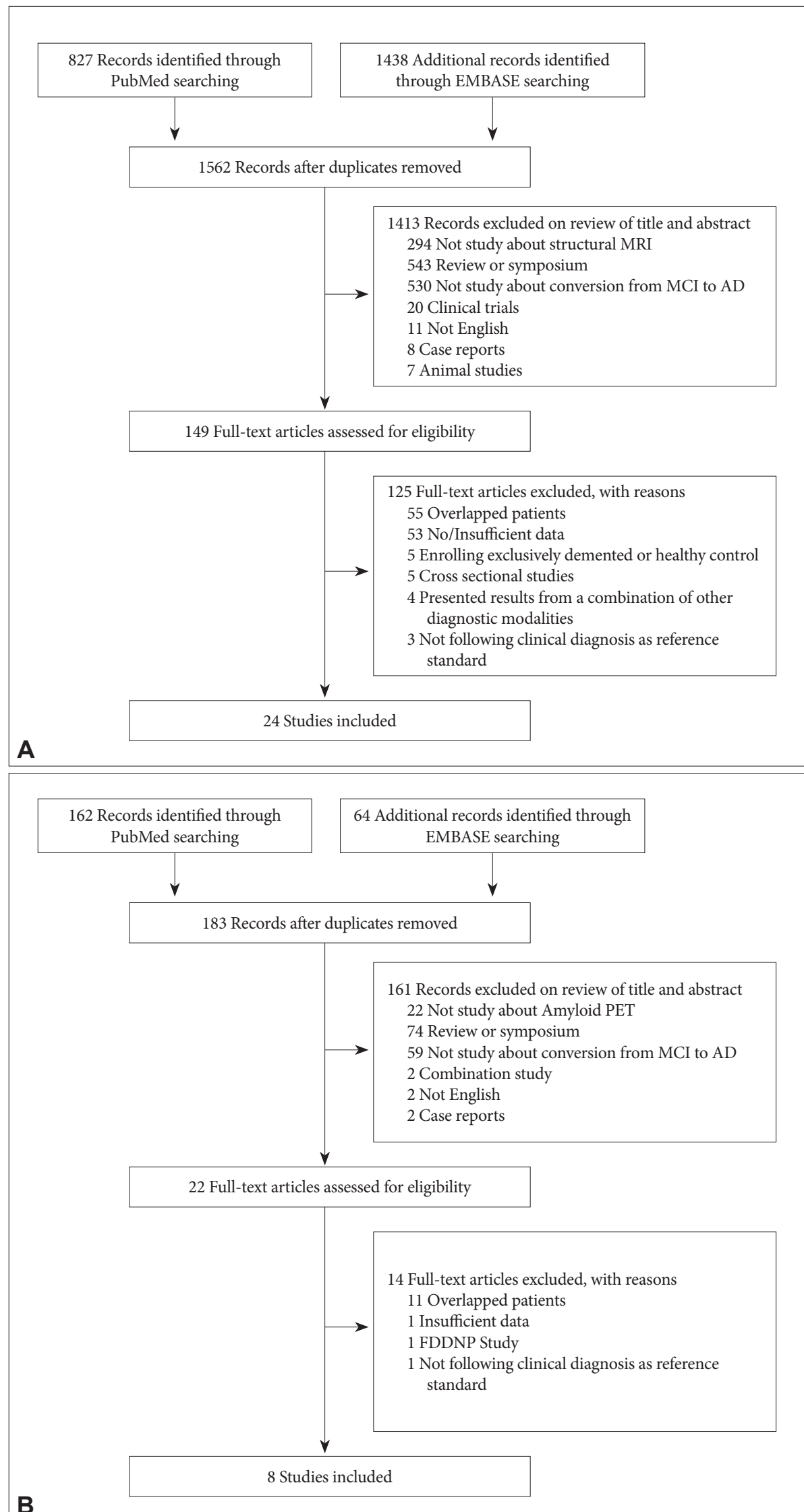

Figure 1. Flow diagram of participant selection for $\mathrm{MCl}$ conversion with structural MRI (A) and amyloid PET (B). 


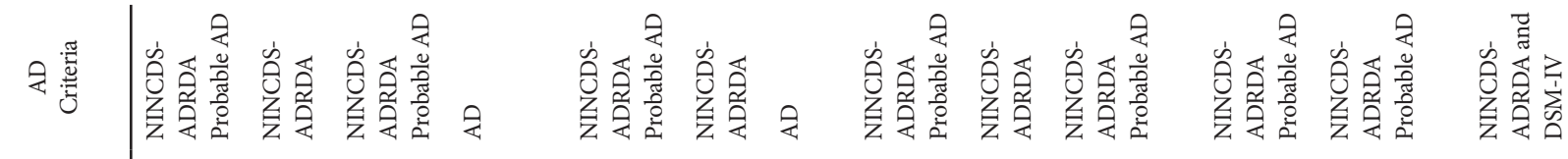

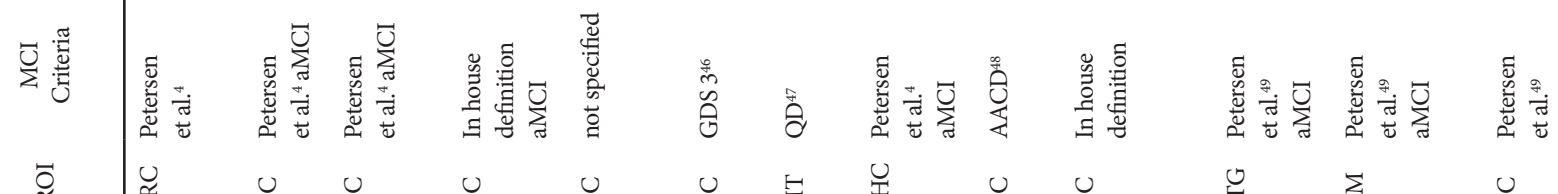

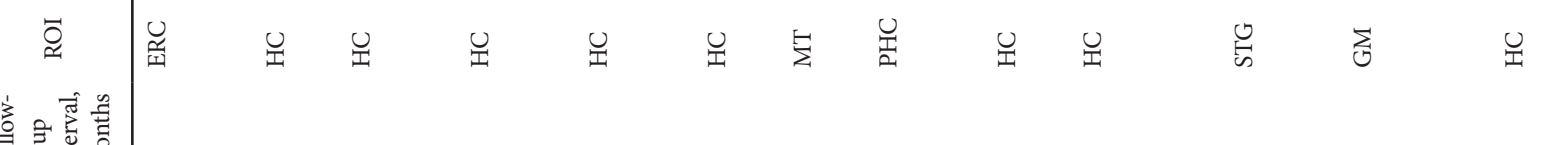

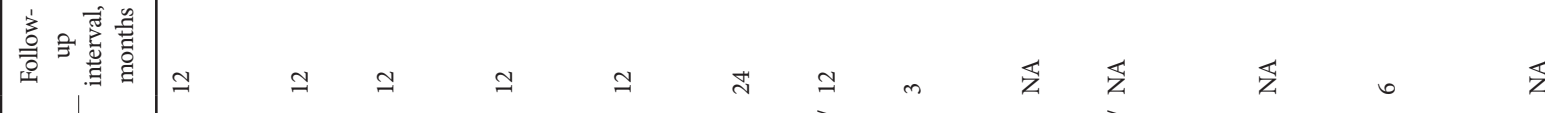

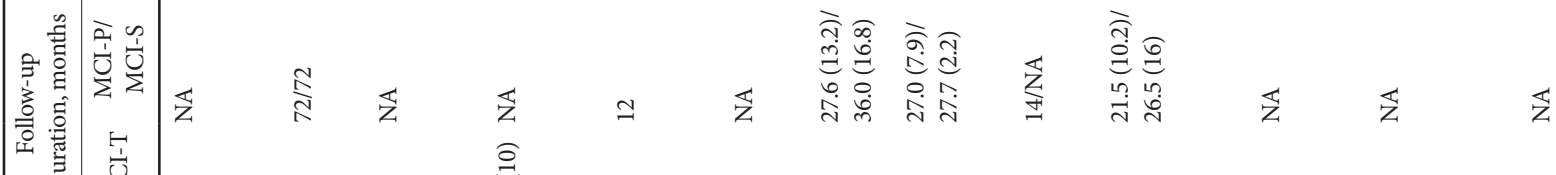

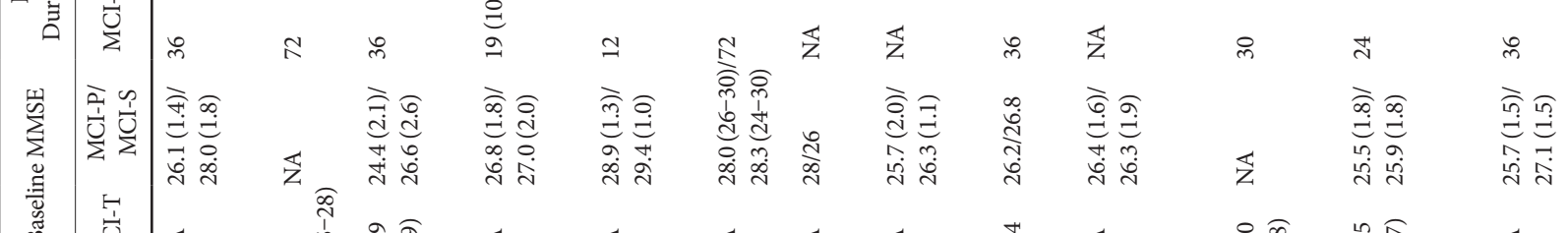

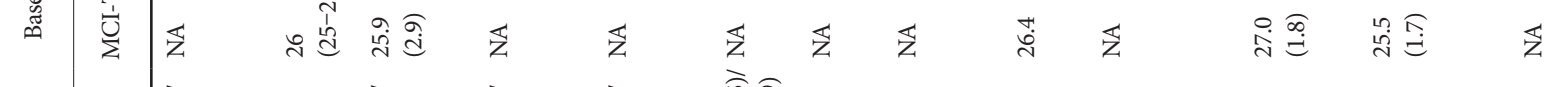

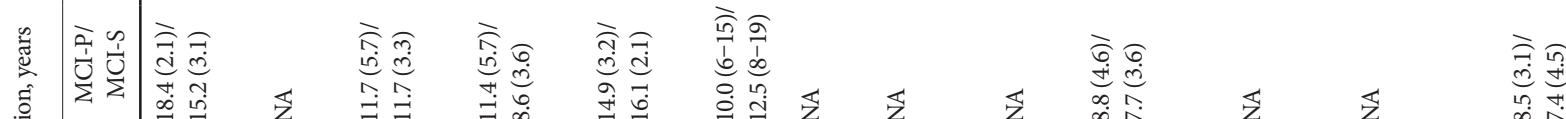

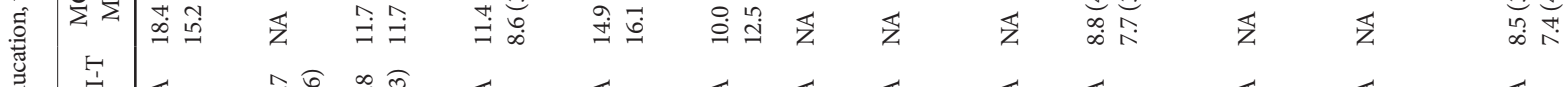

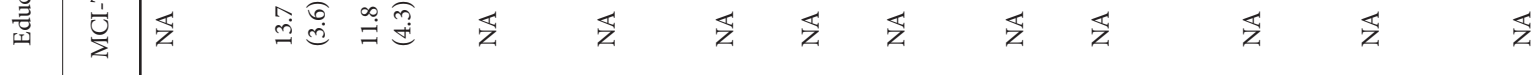

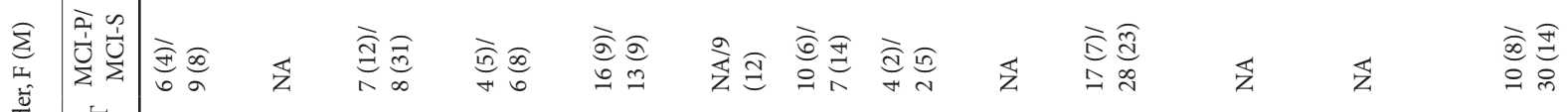

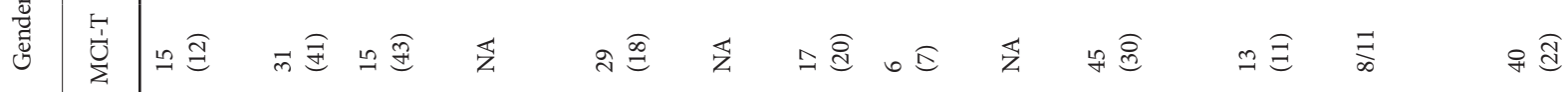

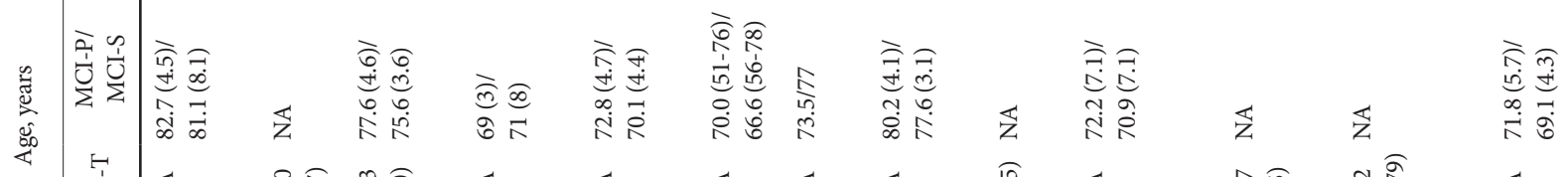

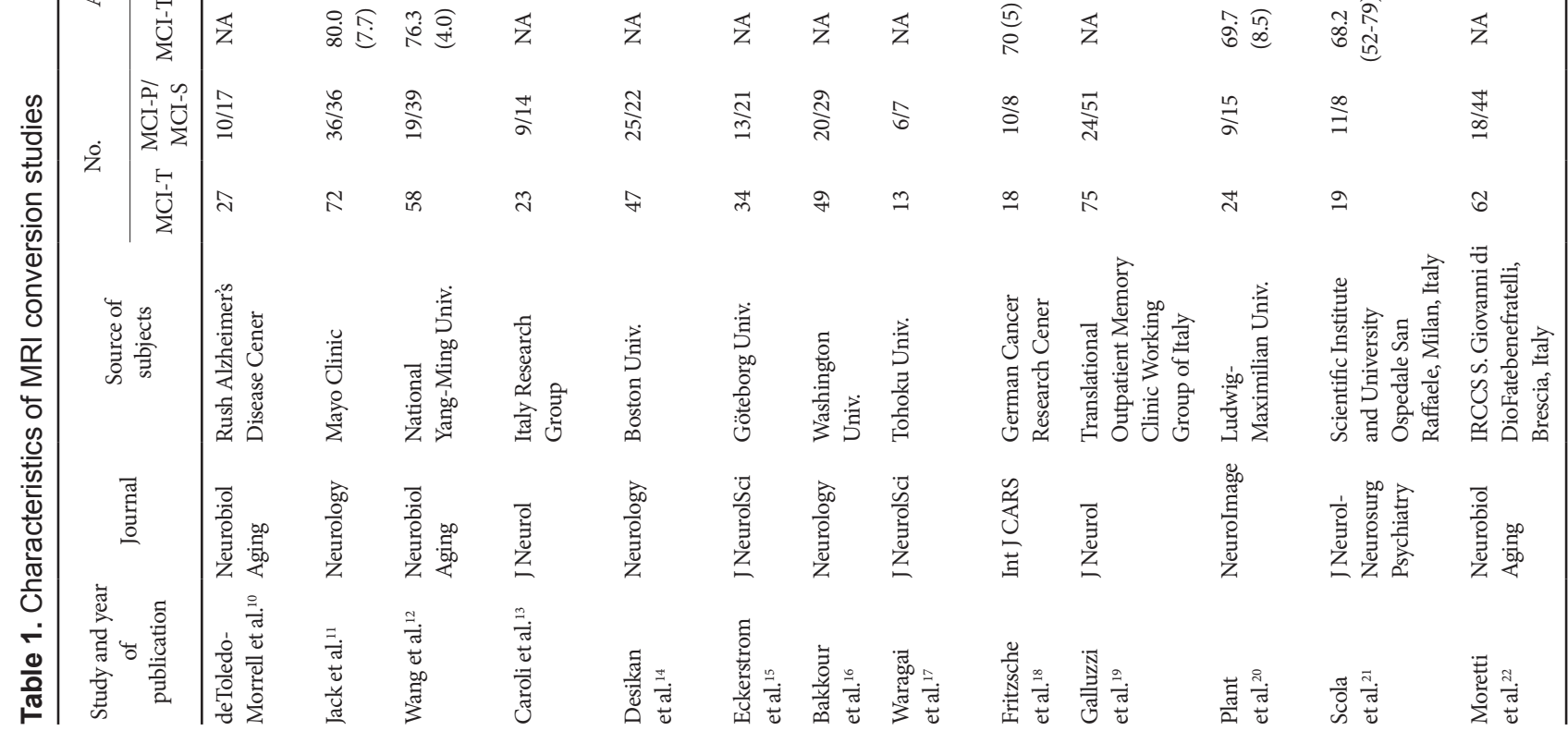




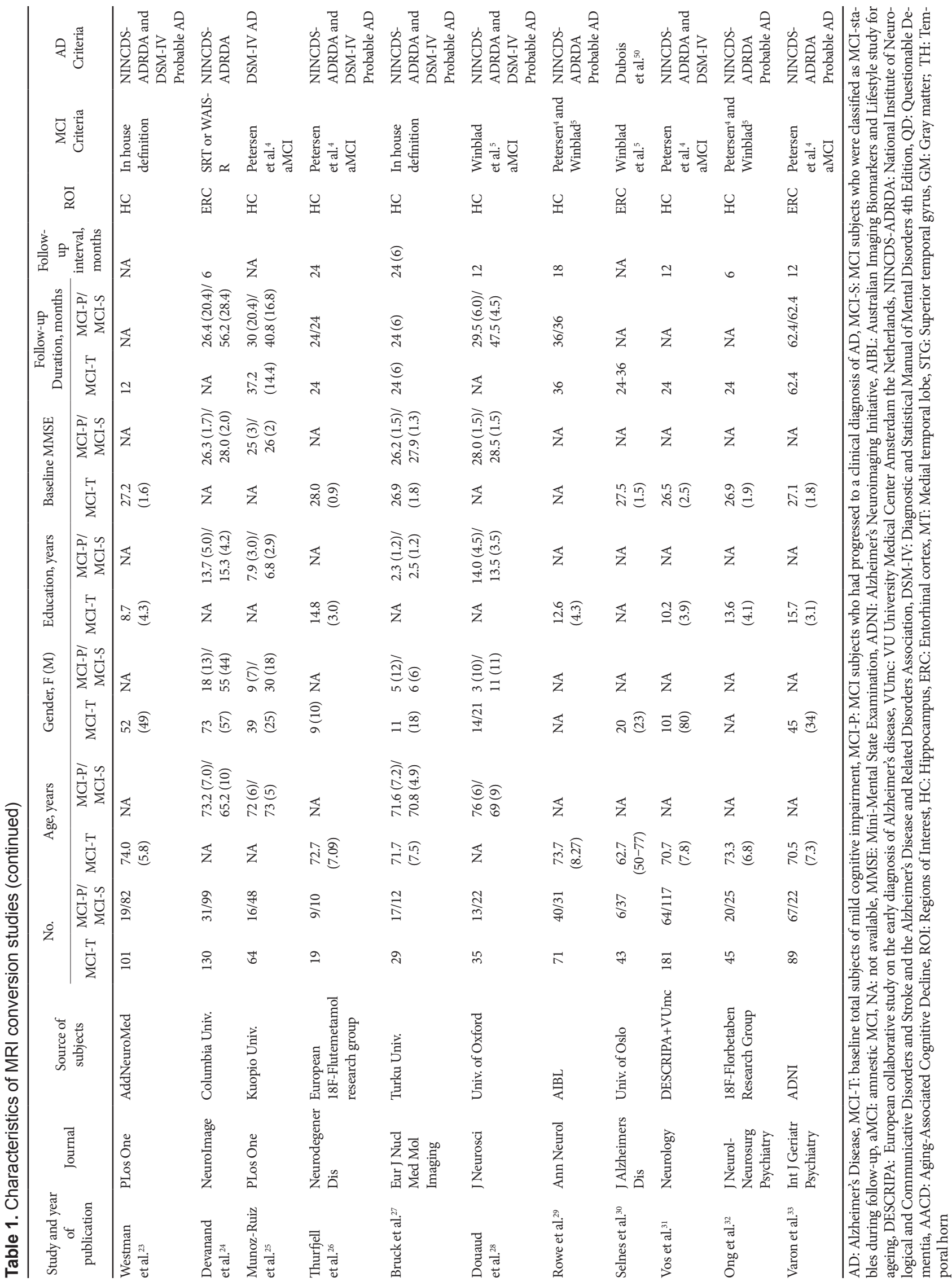


et al., ${ }^{41}$ which included six paper on PIB-PET assessment from 2009 to 2011 , reported pooled sensitivity of $93 \%$ and pooled specificity of $56 \%$ in the prediction of conversion to $\mathrm{AD}$ from MCI. Another recent PIB-PET meta-analysis, which included 8 papers published from 2009 to 2013, identified risk ratios for cognitive progression for cognitively normal, patients with $\mathrm{MCI}$ and patients with $\mathrm{AD}$ group. ${ }^{42}$ Relative risk from a fixedeffects model was 4.03 (95\% CI 2.68-6.07) in patients with MCI. Only one paper in that meta-analysis (Nordberg et al.) overlaps with the papers included in our study. The findings from this previous meta-analysis cannot be compared with the findings of our current study.

Some studies evaluated two or more biomarkers for predicting progression from MCI to AD. ${ }^{3,17,19,27,29-32,43}$ Two studies included in this meta-analysis assessed hippocampus volume by MRI and amyloid burden by PET in the same patients. ${ }^{29,32}$ These studies reported higher odds ratios/positive predictive value/negative predictive value/accuracy for amyloid PET than MRI hippocampus volume reduction on MRI. Another European collaborative study predicted conversion to $\mathrm{AD}$ in patients with MCI using CSF A $\beta 42$ concentration, cortical metabolism by FDG-PET, and MRI hippocampus atrophy. ${ }^{43}$ Their results suggested that progression to $\mathrm{AD}$ in patients with MCI increases with greater biological marker severity. Their recent consecutive publication for the NIA-AA and IWG diagnostic criteria reported that FDG-PET was the best individual predictor for progression to $\mathrm{AD}$. However, the best predictive accuracy was achieved by a combination of amyloidosis and neurodegeneration biomarkers (CSF A 342 and FDG-PET or MRI hippocampus atrophy). ${ }^{3}$ These studies did not use amyloid PET and there may be some differences between amyloid PET and CSF A $\beta 42$. Future studies should compare cerebral amyloid burden using both PET and CSF $\mathrm{A} \beta 42$ for $\mathrm{AD}$ prediction.

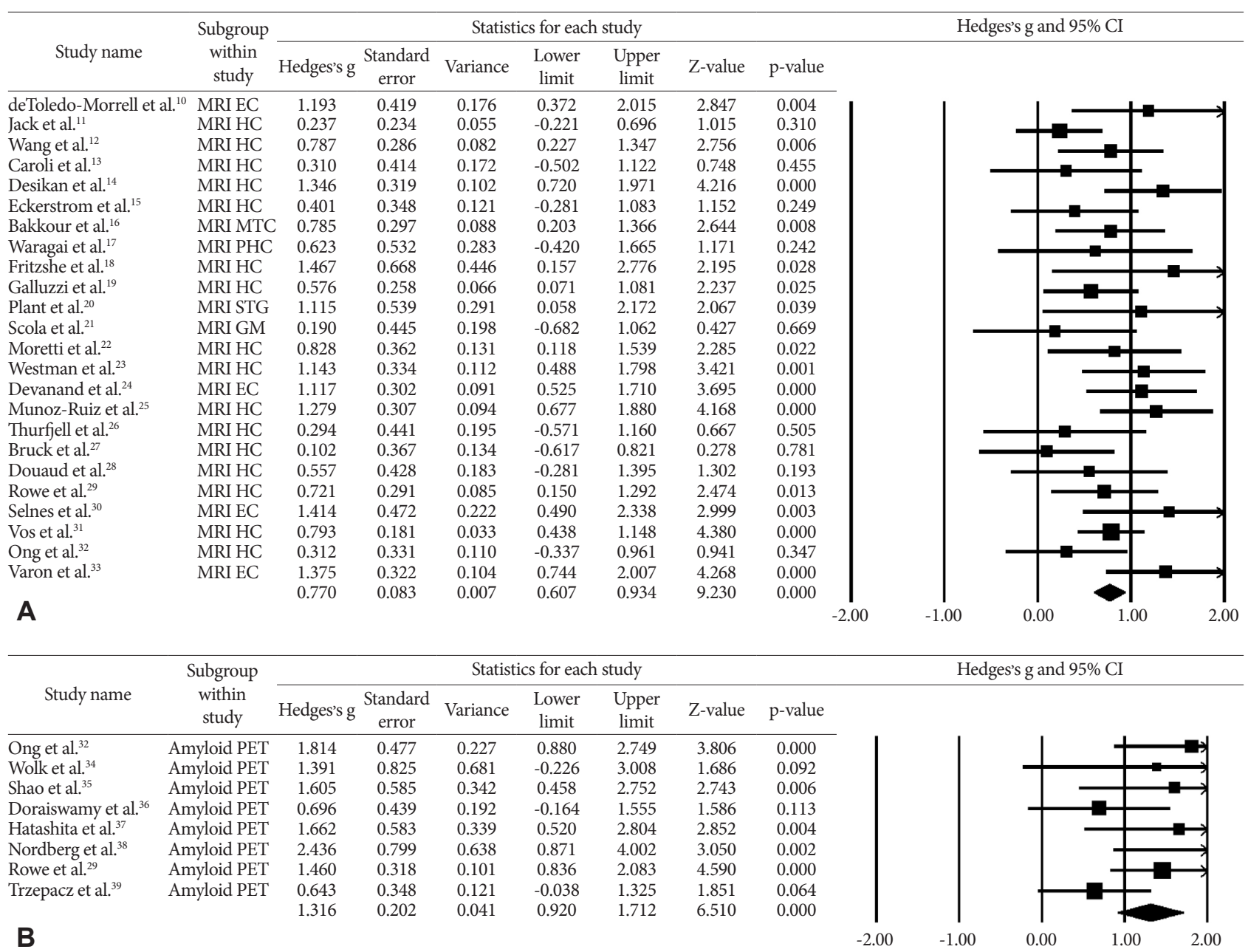

Figure 2. Hedges's g of progressive $\mathrm{MCl}$ versus stable $\mathrm{MCl}$ subjects with clinical follow up (A) Any VOI MRI measurement (B) Amyloid PET (C) Hippocampus volume measurement (D) entorhinal cortex volume measurement. The summary estimates were obtained using a random-effects model. The size of the data markers indicates the weight of the study, which is the inverse of the effect estimate. The diamond data markers indicate the pooled Hedges's g. PHC: parahippocampus cortex, HC: hippocampus, EC: entorhinal cortex, MTC: medial temporal cortex, STG: superior temporal gyrus, GM: gray matter of cerebrum. 
In terms of diagnostic criteria for $\mathrm{MCI}$ and $\mathrm{AD}$, our study applied established criteria for MCI and NINCDS-ADRDA and/or DSM-IV criteria for AD. As indicated in Table 1 and Table 2, 9 studies did not meet proposed MCI criteria for structural MRI assessment ${ }^{13-16,18,19,23,24,27}$ and two studies did not meet criteria for amyloid PET. ${ }^{36,37}$ However, these papers were selected for analysis after the three authors reached the consensus that criteria used were compatible with suggested MCI criteria. Two papers did not indicate the criteria used to diagnosis $\mathrm{AD}^{13,16}$ and one paper used IWG criteria. ${ }^{44}$ Some studies satisfied both NINCDS-ADRDA and DSM-IV criteria, ${ }^{22,23,26-28,31,38}$ and others used only one set of criteria. Some studies that used NINCDS-ADRDA criteria adopted probable $\mathrm{AD},{ }^{10,12,14,17,19-21,23,26-29,32,33}$ whereas other studies did not indicate the diagnosis as probable or possible AD. Our study excluded other types of dementia, not $\mathrm{AD}$.

One limitation of the current meta-analysis was the significant variability in the follow-up interval across studies. Therefore, we cannot exclude the fact that some of the patients with stable MCI may have later developed AD later on. However, results from a previous MRI study in which there was no difference in the duration of follow-up between patients with MCI-P and patients with MCI-S could clearly differentiate differences between the two groups. ${ }^{45}$ This study was not included in our meta-analysis.

The present meta-analysis has several strengths. First, compared with previous studies, we included patients with MCI who progressed to $\mathrm{AD}$ only and studies that applyed homogeneous diagnostic criteria for both MCI and AD. Second, we searched papers within a broad range of time from 2000 to 2014, which increased our sample size for meta-analysis of structural MRI assessments.

Our study also has several limitations. First, the clinical diagnosis of $\mathrm{AD}$ is not always correct; therefore, findings from studies with post-mortem confirmation of $\mathrm{AD}$ diagnosis are more convincing than those from studies with a clinical diagnosis of early-stage $\mathrm{AD}$ among patients with MCI. The studies included in this meta-analysisdid not confirm the clinical diagnosis of AD (or MCI) with neuropathological findings. Second, the follow-up duration was not consistent across studies. Third, patients with MCI were included in the meta-analysis irrespective of clinical phenotype (amnestic/non-amnestic), and patients with $\mathrm{AD}$ were included irrespective of probable/ possible AD. Fourth, subgroup analysis for entorhinal cortex volume and amyloid PET analysis included a small number of studies. Fifth, different research groups used different modalities to assess the level of atrophy with MRI. These modalities include regional volume measures versus cortical thick-

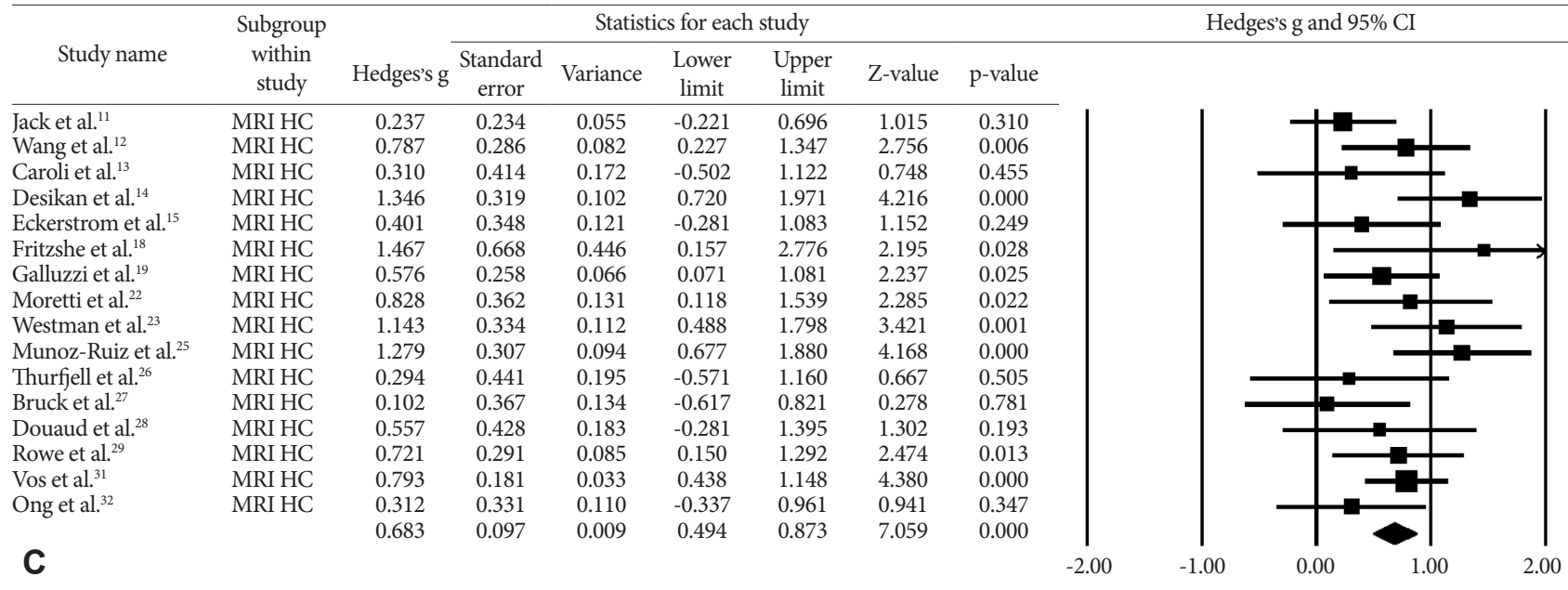

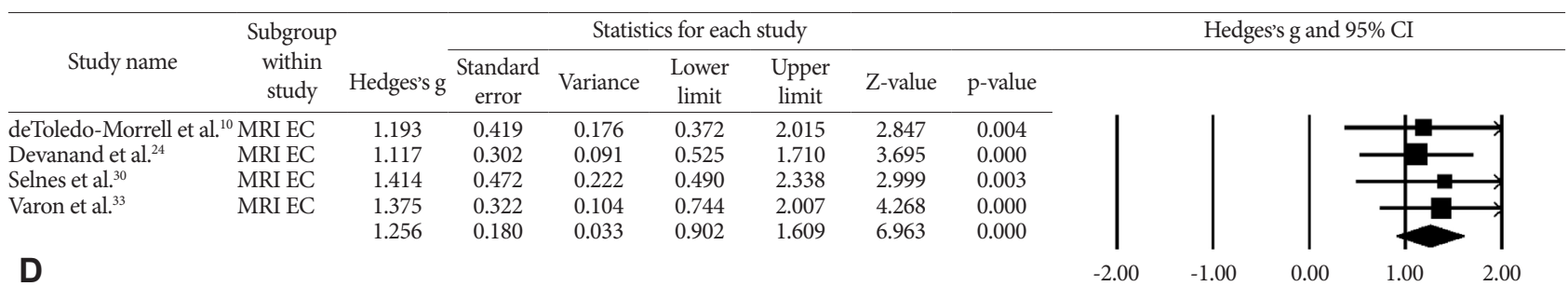

Figure 2. Hedges's g of progressive $\mathrm{MCl}$ versus stable $\mathrm{MCl}$ subjects with clinical follow up (A) Any VOI MRI measurement (B) Amyloid PET (C) Hippocampus volume measurement (D) entorhinal cortex volume measurement. The summary estimates were obtained using a random-effects model. The size of the data markers indicates the weight of the study, which is the inverse of the effect estimate. The diamond data markers indicate the pooled Hedges's g. PHC: parahippocampus cortex, HC: hippocampus, EC: entorhinal cortex, MTC: medial temporal cortex, STG: superior temporal gyrus, GM: gray matter of cerebrum. 


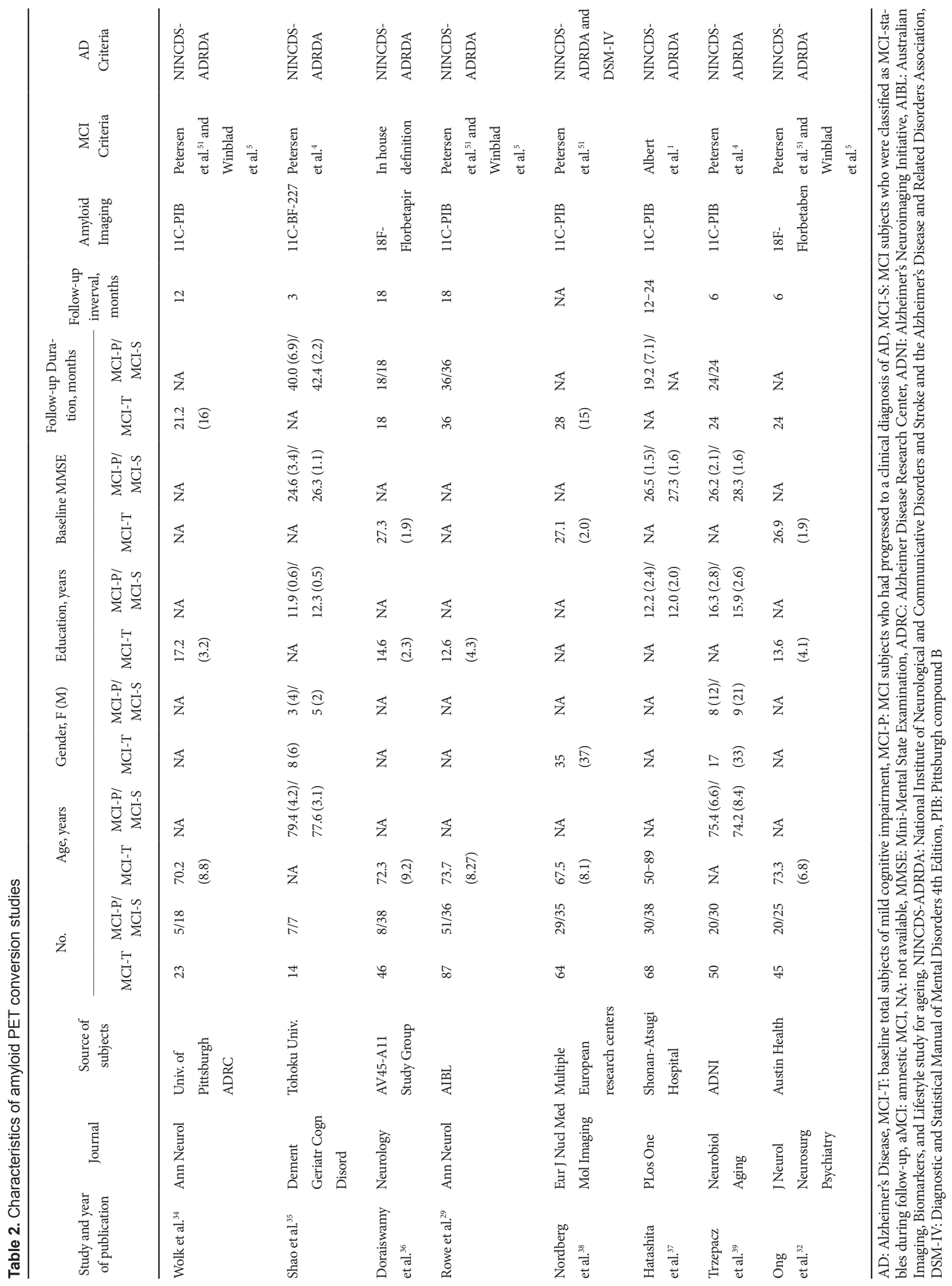


ness and manual volume tracing versus automatic volume tracing. Thresholds for abnormality also vary across laboratories, which increases the error variance in the assignment of normal versus abnormal.

As we know, the heterogeneity and underlying bias is inevitable in most meta-analyses, and may be present in our study. We may have overestimated the predictive accuracy of structural MRI and amyloid PET in the progression to $\mathrm{AD}$ form MCI because some studies included in the meta-analysis dichotomized scores according to the best cut-off in their study.

Our results suggest although amyloid PET is overall a better predictor of progression to AD from MCI than MRI atrophy measures, the entorhinal cortex atrophy measure on MRI is comparable in prediction value to amyloid PET. Data from this meta-analysiscould inform research on clinical applications of MRI volume measurements and be key to the development of guidelines and practice parameters for the use of $\mathrm{AD}$ biomarkers in the clinic.

\section{Supplementary Materials}

The online-only Data Supplement is available with this article at https://doi.org/10.4306/pi.2017.14.2.205.

\section{Acknowledgments}

This work was supported by research fund from a Basic Science Research Program through the National Research Foundation of Korea (NRF) funded by the Ministry of Education (2014R1A1A2054062) and the Brain Research Program through the NRF funded by the Ministry of Science, ICT \& Future Planning (NRF-2014M3C7A1046041).

\section{REFERENCES}

1. Albert MS, DeKosky ST, Dickson D, Dubois B, Feldman HH, Fox NC, et al. The diagnosis of mild cognitive impairment due to Alzheimer's disease: recommendations from the National Institute on Aging-Alzheimer's Association workgroups on diagnostic guidelines for Alzheimer's disease. Alzheimers Dement 2011;7:270-279.

2. Dubois B, Feldman HH, Jacova C, Cummings JL, Dekosky ST, Barberger-Gateau P, et al. Revising the definition of Alzheimer's disease: a new lexicon. Lancet Neurol 2010;9:1118-1127.

3. Prestia A, Caroli A, Wade SK, van der Flier WM, Ossenkoppele R, Van Berckel $\mathrm{B}$, et al. Prediction of $\mathrm{AD}$ dementia by biomarkers following the NIA-AA and IWG diagnostic criteria in MCI patients from three European memory clinics. Alzheimers Dement 2015;11:1191-1201.

4. Petersen RC, Smith GE, Waring SC, Ivnik RJ, Tangalos EG, Kokmen E. Mild cognitive impairment: clinical characterization and outcome. Arch Neurol 1999;56:303-308.

5. Winblad B, Palmer K, Kivipelto M, Jelic V, Fratiglioni L, Wahlund LO, et al. Mild cognitive impairment--beyond controversies, towards a consensus: report of the International Working Group on Mild Cognitive Impairment. J Intern Med 2004;256:240-246.

6. McKhann G, Drachman D, Folstein M, Katzman R, Price D, Stadlan EM. Clinical diagnosis of Alzheimer's disease: report of the NINCDSADRDA Work Group under the auspices of Department of Health and Human Services Task Force on Alzheimer's Disease. Neurology 1984; 34:939-944.

7. American Psychiatric Association. Diagnostic and Statistical Manual of Mental Disorders: DSM-IV (4th Ed.). Washington, DC: American Psychiatric Association; 1994.
8. Whiting PF, Rutjes AW, Westwood ME, Mallett S, Deeks JJ, Reitsma JB, et al. QUADAS-2: a revised tool for the quality assessment of diagnostic accuracy studies. Ann Intern Med 2011;155:529-536.

9. Higgins JP, Thompson SG. Quantifying heterogeneity in a meta-analysis. Stat Med 2002;21:1539-1558.

10. deToledo-Morrell L, Stoub TR, Bulgakova M, Wilson RS, Bennett DA, Leurgans S, et al. MRI-derived entorhinal volume is a good predictor of conversion from MCI to AD. Neurobiol Aging 2004;25:1197-1203.

11. Jack CR Jr, Shiung MM, Weigand SD, O’Brien PC, Gunter JL, Boeve $\mathrm{BF}$, et al. Brain atrophy rates predict subsequent clinical conversion in normal elderly and amnestic MCI. Neurology 2005;65:1227-1231.

12. Wang PN, Lirng JF, Lin KN, Chang FC, Liu HC. Prediction of Alzheimer's disease in mild cognitive impairment: a prospective study in Taiwan. Neurobiol Aging 2006;27:1797-1806.

13. Caroli A, Testa C, Geroldi C, Nobili F, Barnden LR, Guerra UP, et al. Cerebral perfusion correlates of conversion to Alzheimer's disease in amnestic mild cognitive impairment. J Neurol 2007;254:1698-1707.

14. Desikan RS, Fischl B, Cabral HJ, Kemper TL, Guttmann CR, Blacker $\mathrm{D}$, et al. MRI measures of temporoparietal regions show differential rates of atrophy during prodromal AD. Neurology 2008;71:819-825.

15. Eckerstrom C, Olsson E, Borga M, Ekholm S, Ribbelin S, Rolstad S, et al. Small baseline volume of left hippocampus is associated with subsequent conversion of MCI into dementia: the Goteborg MCI study. J Neurol Sci 2008;272:48-59.

16. Bakkour A, Morris JC, Dickerson BC. The cortical signature of prodromal AD: regional thinning predicts mild AD dementia. Neurology 2009;72:1048-1055.

17. Waragai M, Okamura N, Furukawa K, Tashiro M, Furumoto S, Funaki $\mathrm{Y}$, et al. Comparison study of amyloid PET and voxel-based morphometry analysis in mild cognitive impairment and Alzheimer's disease. J Neurol Sci 2009;285:100-108.

18. Fritzsche KH, Stieltjes B, Schlindwein S, van Bruggen T, Essig M, Meinzer HP. Automated MR morphometry to predict Alzheimer's disease in mild cognitive impairment. Int J Comput Assist Radiol Surg 2010;5:623-632.

19. Galluzzi S, Geroldi C, Ghidoni R, Paghera B, Amicucci G, Bonetti M, et al. The new Alzheimer's criteria in a naturalistic series of patients with mild cognitive impairment. J Neurol 2010;257:2004-2014.

20. Plant C, Teipel SJ, Oswald A, Bohm C, Meindl T, Mourao-Miranda J, et al. Automated detection of brain atrophy patterns based on MRI for the prediction of Alzheimer's disease. Neuroimage 2010;50:162-174.

21. Scola E, Bozzali M, Agosta F, Magnani G, Franceschi M, Sormani MP, et al. A diffusion tensor MRI study of patients with MCI and AD with a 2-year clinical follow-up. J Neurol Neurosurg Psychiatry 2010;81: 798-805.

22. Moretti DV, Frisoni GB, Fracassi C, Pievani M, Geroldi C, Binetti G, et al. MCI patients' EEGs show group differences between those who progress and those who do not progress to AD. Neurobiol Aging 2011;32: 563-571.

23. Westman E, Cavallin L, Muehlboeck JS, Zhang Y, Mecocci P, Vellas B, et al. Sensitivity and specificity of medial temporal lobe visual ratings and multivariate regional MRI classification in Alzheimer's disease. PLoS One 2011;6:e22506.

24. Devanand DP, Bansal R, Liu J, Hao X, Pradhaban G, Peterson BS. MRI hippocampal and entorhinal cortex mapping in predicting conversion to Alzheimer's disease. Neuroimage 2012;60:1622-1629.

25. Munoz-Ruiz MA, Hartikainen P, Koikkalainen J, Wolz R, Julkunen V, Niskanen E, et al. Structural MRI in frontotemporal dementia: comparisons between hippocampal volumetry, tensor-based morphometry and voxel-based morphometry. PLoS One 2012;7:e52531.

26. Thurfjell L, Lotjonen J, Lundqvist R, Koikkalainen J, Soininen H, Waldemar G, et al. Combination of biomarkers: PET [18F]flutemetamol imaging and structural MRI in dementia and mild cognitive impairment. Neurodegener Dis 2012;10:246-249.

27. Bruck A, Virta JR, Koivunen J, Koikkalainen J, Scheinin NM, Helenius 
$\mathrm{H}$, et al. [11C]PIB, [18F]FDG and MR imaging in patients with mild cognitive impairment. Eur J Nucl Med Mol Imaging 2013;40:1567-1572.

28. Douaud G, Menke RA, Gass A, Monsch AU, Rao A, Whitcher B, et al. Brain microstructure reveals early abnormalities more than two years prior to clinical progression from mild cognitive impairment to $\mathrm{Al}$ zheimer's disease. J Neurosci 2013;33:2147-2155.

29. Rowe CC, Bourgeat P, Ellis KA, Brown B, Lim YY, Mulligan R, et al. Predicting Alzheimer disease with beta-amyloid imaging: results from the Australian imaging, biomarkers, and lifestyle study of ageing. Ann Neurol 2013;74:905-913.

30. Selnes P, Aarsland D, Bjornerud A, Gjerstad L, Wallin A, Hessen E, et al. Diffusion tensor imaging surpasses cerebrospinal fluid as predictor of cognitive decline and medial temporal lobe atrophy in subjective cognitive impairment and mild cognitive impairment. J Alzheimers Dis 2013;33:723-736.

31. Vos SJ, van Rossum IA, Verhey F, Knol DL, Soininen H, Wahlund LO, et al. Prediction of Alzheimer disease in subjects with amnestic and nonamnestic MCI. Neurology 2013;80:1124-1132.

32. Ong KT, Villemagne VL, Bahar-Fuchs A, Lamb F, Langdon N, Catafau $\mathrm{AM}$, et al. Abeta imaging with $18 \mathrm{~F}$-florbetaben in prodromal Alzheimer's disease: a prospective outcome study. J Neurol Neurosurg Psychiatry 2015;86:431-436.

33. Varon D, Barker W, Loewenstein D, Greig M, Bohorquez A, Santos I, et al. Visual rating and volumetric measurement of medial temporal atrophy in the Alzheimer's Disease Neuroimaging Initiative (ADNI) cohort: baseline diagnosis and the prediction of MCI outcome. Int J Geriatr Psychiatry 2015;30:192-200.

34. Wolk DA, Price JC, Saxton JA, Snitz BE, James JA, Lopez OL, et al. Amyloid imaging in mild cognitive impairment subtypes. Ann Neurol 2009;65:557-568.

35. Shao H, Okamura N, Sugi K, Furumoto S, Furukawa K, Tashiro M, et al. Voxel-based analysis of amyloid positron emission tomography probe [C]BF-227 uptake in mild cognitive impairment and alzheimer's disease. Dement Geriatr Cogn Disord 2010;30:101-111.

36. Doraiswamy PM, Sperling RA, Coleman RE, Johnson KA, Reiman EM, Davis MD, et al. Amyloid-beta assessed by florbetapir F 18 PET and 18-month cognitive decline: a multicenter study. Neurology 2012;79: 1636-1644.

37. Hatashita S, Yamasaki H. Diagnosed mild cognitive impairment due to Alzheimer's disease with PET biomarkers of beta amyloid and neuronal dysfunction. PLoS One 2013;8:e66877.

38. Nordberg A, Carter SF, Rinne J, Drzezga A, Brooks DJ, Vandenberghe $\mathrm{R}$, et al. A European multicentre PET study of fibrillar amyloid in Alzheimer's disease. Eur J Nucl Med Mol Imaging 2013;40:104-114.
39. Trzepacz PT, Yu P, Sun J, Schuh K, Case M, Witte MM, et al. Comparison of neuroimaging modalities for the prediction of conversion from mild cognitive impairment to Alzheimer's dementia. Neurobiol Aging 2014;35:143-151.

40. Yuan Y, Gu ZX, Wei WS. Fluorodeoxyglucose-positron-emission tomography, single-photon emission tomography, and structural MR imaging for prediction of rapid conversion to Alzheimer disease in patients with mild cognitive impairment: a meta-analysis. AJNR Am J Neuroradiol 2009;30:404-410.

41. Zhang S, Han D, Tan X, Feng J, Guo Y, Ding Y. Diagnostic accuracy of 18 F-FDG and 11 C-PIB-PET for prediction of short-term conversion to Alzheimer's disease in subjects with mild cognitive impairment. Int J Clin Pract 2012;66:185-198.

42. Chen X, Li M, Wang S, Zhu H, Xiong Y, Liu X. Pittsburgh compound $\mathrm{B}$ retention and progression of cognitive status--a meta-analysis. Eur J Neurol 2014;21:1060-1067.

43. Prestia A, Caroli A, van der Flier WM, Ossenkoppele R, Van Berckel B, Barkhof F, et al. Prediction of dementia in MCI patients based on core diagnostic markers for Alzheimer disease. Neurology 2013;80:1048-1056.

44. Dubois B, Feldman HH, Jacova C, Dekosky ST, Barberger-Gateau P, Cummings J, et al. Research criteria for the diagnosis of Alzheimer's disease: revising the NINCDS-ADRDA criteria. Lancet Neurol 2007;6: 734-746.

45. Whitwell JL, Shiung MM, Przybelski SA, Weigand SD, Knopman DS, Boeve BF, et al. MRI patterns of atrophy associated with progression to AD in amnestic mild cognitive impairment. Neurology 2008;70:512-520.

46. Reisberg B, Ferris SH, de Leon MJ, Crook T. The Global Deterioration Scale for assessment of primary degenerative dementia. Am J Psychiatry 1982;139:1136-1139.

47. Petersen RC. Mild cognitive impairment or questionable dementia? Arch Neurol 2000;57:643-614.

48. Levy R. Aging-associated cognitive decline. Working Party of the International Psychogeriatric Association in collaboration with the World Health Organization. Int Psychogeriatr 1994;6: 63-68.

49. Petersen RC, Doody R, Kurz A, Mohs RC, Morris JC, Rabins PV, et al. Current concepts in mild cognitive impairment. Arch Neurol 2001;58: 1985-1992.

50. Dubois B, Feldman HH, Jacova C, Dekosky ST, Barberger-Gateau P, Cummings J, et al. Research criteria for the diagnosis of Alzheimer's disease: revising the NINCDS-ADRDA criteria. Lancet Neurol 2007;6:734746.

51. Petersen RC. Mild cognitive impairment as a diagnostic entity. J Intern Med 2004;256:183-194. 